\title{
Triangle Characters of Electrocardiogram for Distinguishing States between Exercise and Relaxation
}

\author{
Yanjun Li, Hong Yan, Jinzhong Song, Xinming Yu, Zongxiao Sun, Hua Wei \\ China Astronaut Research and Training Center, Beijing, China \\ Email: ************
}

Received January 2013

\begin{abstract}
Will exercise-induced cardiovascular workload be monitored by Electrocardiogram (ECG) waveform morphology? The discrimination ability of ECG morphology from 30 subjects was tested for distinguishing states between exercise and relaxation in terms of side lengths, lengths of high lines, angles, perimeters and areas of triangle QRS and triangle T. As a result, 4 characters from triangle QRS had significant differences ( $t$ test, $p<0.05$ ) for over $85 \%$ of subjects in distinguishing between exercise states and relaxation states, which were: ratio of QR side length to RS side length in triangle QRS, angle S and angle Q, as well as the ratio between them. Moreover, ratio of angle $\mathrm{S}$ to angle $\mathrm{Q}$ had significant differences ( $t$ test, $\mathrm{p}<0.05$ ) for all subjects. In conclusion, triangle characters in ECG could be used to distinguish exercise states from relaxation states.
\end{abstract}

Keywords: Electrocardiogram (ECG); Exercise ECG; Exercise-Induced ECG Changes; Triangle QRS; Triangle T

\section{Introduction}

Physical stress during exercise is often monitored by diversified biomedical signals, e.g., Photoplethysmogram (PPG), Electromyogram (EMG), respiratory frequency, blood pressure (BP), Phonocardiogram (PCG), Electrocardiogram (ECG), echocardiogram. And heart rate (HR), heart rate variability (HRV) and systolic blood pressure (SBP) are often considered to be the most popular indices nowadays used in monitoring the activity of physical stress. Activity of parasympathetic (vagal), activity of sympathetic, and the balance between them reveal the effects of stress level on autonomic nervous system (ANS). Parasympathetic tone predominates during relaxation period, sympathetic tone predominates during exercise period which increases the cardiovascular workload and hemodynamic stress (increases HR and SBP while reduces HRV), while parasympathetic tone re-predominates during the recovery period which decreases myocardial function and reduced exercise capacity (reduces HR and SBP while increases HRV) [1]. In the range of physiological investigations available, measurements of HR and HRV which could be derived from heart-rooted signals (e.g. ECG, PCG and PPG), still form the most non-invasive tool for monitoring the effectiveness of stress on ANS activity [2]. Exercise ECG or stress ECG is mostly used to evaluate exercise capacity and detect exercise-induced myocardial ischemia (MI) or assess the presence and severity of coronary artery disease (CAD)
[3], which is more direct and less expensive than other ways such as imaging technology.

However, most researchers focused on detecting MI or CAD by exercise ECG, while the changes in ECG morphological characters have been analyzed less intensively in healthy persons in exercise states. Normal changes in ECG that induced by exercise are usually functional or physiological, and are not associated with cardiac pathological changes and have no clinical effects. Both mental and physical stress increases sympathetic activity and decreases parasympathetic activity [2], which is easily reflected by HR and HRV: HR increases while HRV decreases under stress compared with relaxation [2]. However, more information may be contained in ECG waveform morphology than the ECG rhythm (HR and HRV) [4]. Because of that, variables from morphology may provide incremental value to conventional analysis for detecting exercise-induced ECG changes.

We made the definition of Electrocardio-waveform variability (ECWV) [4] and achieved ECWV quantitative analysis by waveform parameters of correlation coefficient, indices of Karhunen-Loève Transform (KLT), and indices of tessellated map (TM). ECWV had been proved efficient because some indices could distinguish between mental load and non-load states [4]. Yan et al. [5] indicated that the ECG waveforms were more stable in rest states than in stress states based on KLT indices, and proved that KLT indices were suitable to distinguish the states between rest and stress. Triangle characters of 
ECG make the ECG morphology analysis very easy and simple. Song et al. [6] for the first time used barycenters of QRS triangles to remove baseline wandering. Then, we found that triangle characters of ECG were also useful in detecting QRS complex [7]. This paper focused on finding out some ECG waveform indices by triangle character analysis which was useful in distinguishing states between exercise and relaxation, and in monitoring exercise-induced cardiovascular workload.

\section{Methods}

\subsection{Subjects, Assignment and ECG Recording}

Totally 30 healthy subjects (all males, age $28.9 \pm 4.2$ years) participated in the investigation. All subjects reported in good health, and none had taken any forms of drastic activity in the last week. Common exercise modalities used in North America and Australasia are the treadmill exercise test (TET), and stationary bicycle ergometer in Europe [1]. In this paper, stationary bicycle ergometer was used to put workload on subjects. In order to let subjects experience physical stress and relaxation, a work-rest schedule including stationary bicycle exercise and relaxation was specially designed, which was consisted of three sections: resting, physical workload and recovering, and they were described in detail as follows: 1) baseline period: rested on bicycle ergometry for about 5 min; 2) workload period: exercised on bicycle ergometry for about $20 \mathrm{~min}$; 3) recovery period: rested on bicycle ergometry for about $5 \mathrm{~min}$.

During workload period, workload of $100 \mathrm{~W}$ was imposed on each subject by the bicycle ergometry. Each work cycle should be completed nearly at a constant speed, in which subjects trampled on the pedal up and down reduplicatively to ride the stationary bicycle for about $20 \mathrm{~min}$. One channel ECG was continuously recorded by the non-invasive cardiac function monitor through the experiment for each subject. The channel was placed near the V5 precordial position with $\mathrm{Ag} / \mathrm{AgCl}$ electrodes in order to yield large $\mathrm{R}$ wave signals, which was very useful for accurately heartbeat segmentation. ECG signals were sampled with 12 bits A/D converter, and the sampling frequency was $250 \mathrm{~Hz}$.

\subsection{ECG Preprocessing}

ECG collected from body surface is always interfered by noises of different types, especially baseline shifts, postural changes, electrode interference, EMG and power line interference, etc. Noise suppression at the cost of minimum distortions to the desired ECG component plays an important role in ECG pre-processing. In this paper, wavelet transform were used to remove EMG interference, then baseline wandering from ECG was re- moved by wavelet transform combined with QRS barycenter fitting [6].

QRS complex detection usually provides the fundamentals to automated ECG analysis. However, detection of QRS complex is not easy due to abormal shapes, noise, and artifacts. $\mathrm{R}$ waves and $\mathrm{T}$ waves should normally be upright in leads I, II and V3 to V6 [1]. The combination of matched filtering and triangle character analysis [7] was applied for QRS complex detection, which was able to identify QRS complexes reliably even under the condition of poor signal quality [7]. According to the position of R wave peak "Rp", Q wave peak "Qp" and S wave peak "Sp" were located by finding the local minimal val- ues backward and forward from $\mathrm{R}$ peak, in regions of [Rp $-0.1 \mathrm{~s}, \mathrm{Rp}]$ and $[\mathrm{Rp}, \mathrm{Rp}+0.1 \mathrm{~s}]$, respectively.

$\mathrm{T}$ wave end "Tn" was located by the method through the computation of an indicator related to the area covered by the $\mathrm{T}$ wave curve, which was not only computationally simple but also very robust to acquisition noise [8]. Then $T$ wave peak "Tp" was automatically detected by finding the local maximal value in regions of $[R p+$ $0.1 \mathrm{~s}, \mathrm{Tn}]$. However, location of $\mathrm{T}$ wave start point is one of the most difficult problems for ECG waveform boundary location due to the slow transition from ST segment to $\mathrm{T}$ wave start point, or due to the corruption from noise. Instead of locating the precise position for $\mathrm{T}$ wave start point, a point " $\mathrm{Tb}$ " is defined as a rough point of $\mathrm{T}$ wave begin from the left segment of $\mathrm{T}$ wave to "Tp" whose time length is the same as the time span between "Tp" and "Tn" by Equation (1).

$$
\mathrm{Tb}=2 \mathrm{Tp}-\mathrm{Tn}
$$

\subsection{Extraction of ECG Triangle Characters}

A triangle is defined by setting the onset point, the peak point and the offset point of a wave as three apexes of a triangle. A typical triangle extracted from a signal is shown in Figure 1, in which the morphology of a wave is simplified as a triangle $A B C$ [7]. Horizontal distance between apex $A$ and apex $B$ is defined as TmAB by Equation (2). Amplitude AmAB between apex A and apex B in vertical coordinate is gotten by Equation (3).

$$
\mathrm{TmAB}=(\mathrm{Bx}-\mathrm{Ax}) / \mathrm{Fs}
$$

where $\mathrm{Ax}$ and $\mathrm{Bx}$ are $\mathrm{x}$-coordinates of point "A" and point "B" respectively, whose unit are sampling points; Fs is the ECG sampling frequency whose unit are sampling points per second. As a result, unit of TmAB is second.

$$
\mathrm{AmAB}=\mathrm{By}-\mathrm{Ay}
$$

where $\mathrm{Ay}$ and By are $\mathrm{y}$-coordinates of point " $\mathrm{A}$ " and point " $\mathrm{B}$ " respectively, whose unit is millivolt. As a result, unit of AmAB is also millivolt.

The side length of $A B$ in triangle $A B C$, called $S d A B$, 


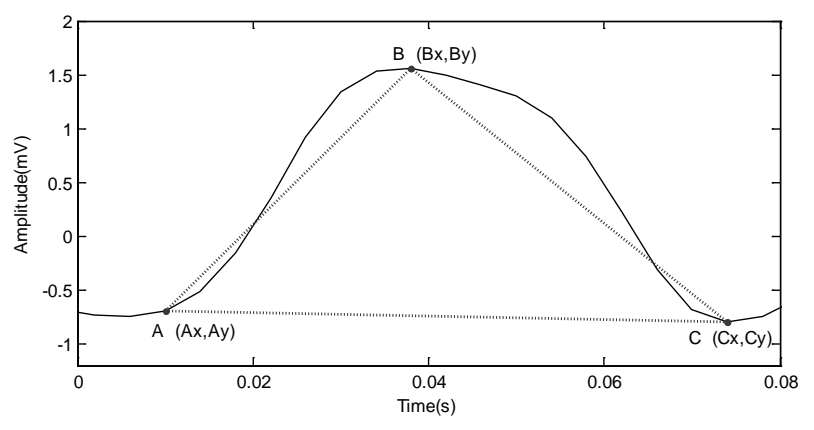

Figure 1. A typical triangle ABC extracted from a signal.

depends on the ECG paper speed in horizon and the amplitude unit in vertical, i.e. side length depends on the time resolution and amplitude resolution. The paper for ECG recording is fully composed of small squares vertically and horizontally with side length as $1 \mathrm{~mm}$. In horizontal orientation, paper moving speed is mostly 25 $\mathrm{mm} / \mathrm{s}$ (paper speed could be adjust to $50 \mathrm{~mm} / \mathrm{s}, 100 \mathrm{~mm} / \mathrm{s}$ or $200 \mathrm{~mm} / \mathrm{s}$ on demand for higher time resolution), while the ratio of amplitude to voltage is mostly 10 $\mathrm{mm} / \mathrm{mV}$ in vertical orientation. For instance, at an ECG paper speed of $200 \mathrm{~mm} / \mathrm{s}$ in horizon and amplitude unit of $10 \mathrm{~mm} / \mathrm{mV}, 1 \mathrm{~mm}$ represents $0.005 \mathrm{~s}$ for $\mathrm{x}$ coordinates while $0.1 \mathrm{mV}$ for y coordinates. Because of that, scales of time and amplitude must be transferred to unit of $\mathrm{mm}$. The side length SdAB is calculated by Equation (4).

$$
S d A B=\sqrt{\left(T m A B / F_{T m}\right)^{2}+\left(A m A B / F_{A m}\right)^{2}}
$$

where $F_{T m}$ is a factor of x-coordinates depending on the paper speed during ECG recording, which could be any value of $\{0.04,0.02,0.01,0.005\} \mathrm{s} / \mathrm{mm} ; F_{A m}$ is a factor of $\mathrm{y}$-coordinates depending on the amplitude resolution, which is often taken as $0.1 \mathrm{mV} / \mathrm{mm}$.

The side length of $\mathrm{AC}$ and $\mathrm{BC}$ in triangle $\mathrm{ABC}$ that called SdAC and SdBC respectively, are obtained with the same way of defining SdAB. Then the perimeter Pm and area Ar of triangle ABC are acquired by Equation (5) and (6), respectively. The high line on side $A B$ that called LnAB in triangle $\mathrm{ABC}$ is gotten by Equation (7). The Angle A called AgA in triangle ABC is obtained according to the law of cosines, as shown by Equation (8).

$$
\begin{gathered}
\mathrm{Pm}=(\mathrm{SdAB}+\mathrm{SdBC}+\mathrm{SdAC}) / 2 \\
A r=\sqrt{\mathrm{P}(\mathrm{P}-\mathrm{SdAB})(\mathrm{P}-\mathrm{SdBC})(\mathrm{P}-\mathrm{SdAC})}
\end{gathered}
$$

where $\mathrm{P}=\mathrm{Pm} / 2$.

$$
\begin{gathered}
\operatorname{LnAB}=2 \mathrm{Ar} / \mathrm{SdAB} \\
\operatorname{Ag} A=\operatorname{ar} \cos \left(\left(b^{2}+c^{2}-a^{2}\right) /(2 b c)\right)
\end{gathered}
$$

where $a, b$, and $c$ represents SdBC, SdAC and SdAB separately; "arcos" is the mathematical operator of arc cosine.
If Qp, Rp and Sp were used as apexes of A, B and C, respectively, then the triangle QRS was constructed, as shown in Figure 2. In the same way, the triangle $\mathrm{T}$ was formed based on the feature points of $\mathrm{Tb}, \mathrm{Tp}$ and $\mathrm{Tn}$.

Totally 26 triangular indices were used for distinguishing states between exercise and relaxation, including 13 indices of triangle QRS and 13 indices of triangle $\mathrm{T}$, as shown in Table 1. In Table 1, "Q", “R”, and "S" represents Q peak, R peak and S peak in QRS complex, respectively; “Tb”, “Tp” and “Tn” represents the beginning, the peak and the end point of $T$ wave, respectively. And $\mathrm{R}-\mathrm{R}$ interval is defined as TmRR to make a comparison with these triangular indices.

\subsection{Comparison between Rest and Workload States}

To make the comparison more clearly among the baseline period, the workload period and the recovery period, three short time periods were picked up from them respectively: 2 min from the beginning of baseline period (P1); 2 min before the peak of exercise period (P2), where exercise peak was defined at the instant of maximal heart rate [9] (see Figure 3); and $2 \mathrm{~min}$ before the end of recovery period (P3).

When testing the significant differences for all subjects between rest and workload states, the difference of vectors were compared with vector "zeros", by which the comparison between relative difference values and vector "zeros" took the place of the comparison between individual values and some objective criterion [2], which
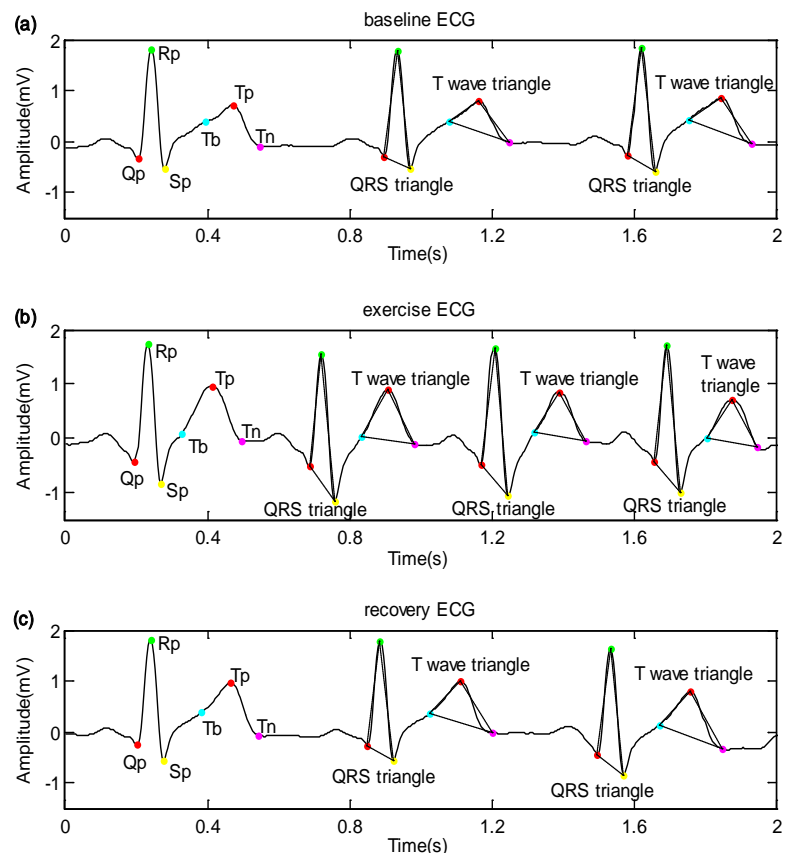

Figure 2. Triangle $Q R S$ based on apexes of $Q p, R p$ and $S p$, and triangle $T$ based on apexes of $T b, T p$ and $T n$. 
Table 1. Definition of ECG measurements.

\begin{tabular}{|c|c|c|}
\hline \multicolumn{3}{|c|}{ (a) Indices of triangle QRS } \\
\hline 1 & SdRS & RS side length in triangle QRS \\
\hline 2 & SdQS & QS side length in triangle QRS \\
\hline 3 & SdQR & QR side length in triangle QRS \\
\hline 4 & SdQR/SdRS & Ratio of SdQR to SdRS \\
\hline 5 & AgQ & Angle Q in triangle QRS \\
\hline 6 & AgR & Angle R in triangle QRS \\
\hline 7 & AgS & Angle $\mathrm{S}$ in triangle QRS \\
\hline 8 & AgS/AgQ & Ratio of AgS to AgQ \\
\hline 9 & PmTirQRS & Perimeter of triangle QRS \\
\hline 10 & ArTirQRS & Area of triangle QRS \\
\hline 11 & LnRS & High line on SdRS of triangle QRS \\
\hline 12 & LnQS & High line on SdQS of triangle QRS \\
\hline 13 & LnQR & High line on SdQR of triangle QRS \\
\hline \multicolumn{3}{|c|}{ (b) Indices of triangle $T$} \\
\hline 1 & SdTpTn & TpTn side length in triangle $T$ \\
\hline 2 & SdTbTn & TbTn side length in triangle $T$ \\
\hline 3 & SdTbTp & TbTp side length in triangle $T$ \\
\hline 4 & SdTbTp/SdTpTn & Ratio of SdTbTp to SdTpTn \\
\hline 5 & $\mathrm{AgTb}$ & Angle $\mathrm{Tb}$ in triangle $\mathrm{T}$ \\
\hline 6 & AgTp & Angle $\mathrm{Tp}$ in triangle $\mathrm{T}$ \\
\hline 7 & AgTn & Angle $\mathrm{Tn}$ in triangle $\mathrm{T}$ \\
\hline 8 & AgTn/AgTb & Ratio of AgTn to AgTb \\
\hline 9 & PmTirTw & Perimeter of triangle $\mathrm{T}$ \\
\hline 10 & ArTirTw & Area of triangle $\mathrm{T}$ \\
\hline 11 & LnTpTn & High line on SdTpTn in triangle $\mathrm{T}$ \\
\hline 12 & LnTbTn & High line on SdTbTn in triangle T \\
\hline 13 & LnTbTp & High line on SdTbTp in triangle $\mathrm{T}$ \\
\hline
\end{tabular}

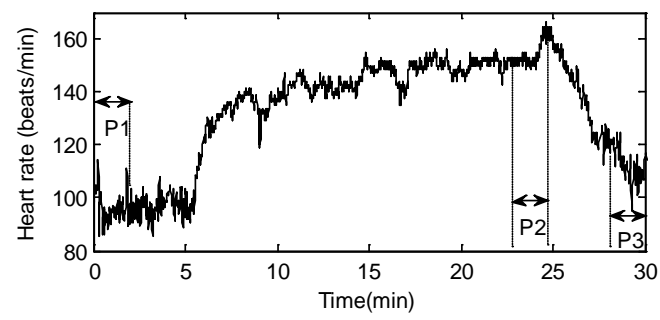

Figure 3. Time periods extraction.

made the influence of the subject"s individual difference weaker and the comparison results more believable. When testing the significant difference between rest and workload states for individual subject, vectors during period P1 and those during period P2 were compared, and vectors during period $\mathrm{P} 2$ and those during period $\mathrm{P} 3$ were compared. At last, the percent of the subjects for which indices had significant difference individually between rest and workload was statistically analyzed.

\section{Results}

\subsection{Comparison between Rest and Workload States}

After the stages of noise suppression, feature point location and ECG characters extraction, the mean value and standard deviation of all indices from all subjects between periods of $\mathrm{P} 1$ and $\mathrm{P} 2$, and that between periods of P2 and P3 were obtained and shown in Table 2. As a result, 10 indices except for TmRR had significant difference (SPSS 17.0 for t test, $\mathrm{P}<0.05$ ) not only between baseline period (P1) and exercise period (P2), but also between exercise period (P2) and recovery period (P3), which were: SdQS, SdQR/SdRS, AgQ, AgS, AgS/AgQ, LnQR, SdTpTn, PmTirTw, ArTirTw, and LnTbTp. These 10 indices had the potential values as stress level

Table 2. Indices variation between rest and exercise.

\begin{tabular}{|c|c|c|c|}
\hline No & Index & $\triangle \mathrm{P} 1 \mathrm{P} 2$ & $\triangle \mathrm{P} 2 \mathrm{P} 3$ \\
\hline 1 & TmRR & $-0.1900 \pm 0.0862^{* * *}$ & $0.0926 \pm 0.0579^{\# \# \#}$ \\
\hline 2 & SdRS & $0.2468 \pm 0.6078$ & $-0.3793 \pm 0.4667^{\# \#}$ \\
\hline 3 & SdQS & $0.1052 \pm 0.1461^{* *}$ & $-0.1168 \pm 0.1493^{\# \#}$ \\
\hline 4 & SdQR & $-0.2983 \pm 0.5895$ & $-0.0689 \pm 0.4144$ \\
\hline 5 & SdQR/SdRS & $-0.1077 \pm 0.0672^{* * *}$ & $0.0512 \pm 0.0507^{\# \#}$ \\
\hline 6 & AgQ & $17.8850 \pm 10.1098^{* * *}$ & $-10.2551 \pm 8.3149^{\# \# \#}$ \\
\hline 7 & AgR & $-2.1481 \pm 4.3544$ & $2.6471 \pm 4.0557^{\#}$ \\
\hline 8 & AgS & $-15.7458 \pm 9.4252^{* * * *}$ & $7.6169 \pm 8.0450^{\# \# \#}$ \\
\hline 9 & AgS/AgQ & $-0.2858 \pm 0.1821^{* * *}$ & $0.1328 \pm 0.1203^{\# \# \#}$ \\
\hline 10 & PmTirQRS & $0.0537 \pm 1.1188$ & $-0.5650 \pm 0.8940^{\#}$ \\
\hline 11 & ArTirQRS & $-0.1894 \pm 0.4726$ & $-0.1297 \pm 0.3893$ \\
\hline 12 & LnRS & $-0.1622 \pm 0.1371^{* * *}$ & $0.0505 \pm 0.1192$ \\
\hline 13 & LnQS & $-0.4760 \pm 0.7386^{*}$ & $0.0873 \pm 0.5054$ \\
\hline 14 & LnQR & $0.0259 \pm 0.0402^{*}$ & $-0.0455 \pm 0.0828^{\#}$ \\
\hline 15 & SdTpTn & $-0.3772 \pm 0.3649^{* * *}$ & $0.3302 \pm 0.3425^{\# \# \#}$ \\
\hline 16 & SdTbTn & $-0.3780 \pm 0.4164^{* *}$ & $0.1305 \pm 0.2617$ \\
\hline 17 & SdTbTp & $-0.0354 \pm 0.2581$ & $0.2292 \pm 0.4161^{\#}$ \\
\hline 18 & SdTbTp/SdTpTn & $0.1161 \pm 0.1150^{* * *}$ & $-0.0325 \pm 0.0795$ \\
\hline 19 & $\mathrm{AgTb}$ & $-2.7229 \pm 11.2287$ & $4.8310 \pm 7.3332^{\#}$ \\
\hline 20 & AgTp & $-2.2021 \pm 15.5652$ & $-7.5891 \pm 12.7279^{\#}$ \\
\hline 21 & AgTn & $4.9009 \pm 6.8621^{* *}$ & $2.7896 \pm 7.0906$ \\
\hline 22 & $\mathrm{AgTn} / \mathrm{AgTb}$ & $0.1457 \pm 0.1601^{* *}$ & $-0.0404 \pm 0.1094$ \\
\hline 23 & PmTirTw & $-0.7877 \pm 0.8161^{* * *}$ & $0.6892 \pm 0.9005^{\# \#}$ \\
\hline 24 & ArTirTw & $-0.3991 \pm 0.4928^{* *}$ & $0.5692 \pm 0.7469^{\#}$ \\
\hline 25 & LnTpTn & $-0.0404 \pm 0.2991$ & $0.1918 \pm 0.3439^{\#}$ \\
\hline 26 & LnTbTn & $-0.0885 \pm 0.3046$ & $0.3112 \pm 0.4163^{\#}$ \\
\hline 27 & LnTbTp & $-0.3730 \pm 0.3644^{* * *}$ & $0.2934 \pm 0.2686^{\# \# \#}$ \\
\hline
\end{tabular}

Note: ${ }^{* * *} \mathrm{p}<0.001,{ }^{* *} \mathrm{p}<0.01,{ }^{*} \mathrm{p}<0.05$, as relative difference values $\triangle \mathrm{P} 1 \mathrm{P} 2$ compared with vector "zeros"; ${ }^{\prime \prime} \mathrm{p}<0.001$, ${ }^{\# \#} \mathrm{p}<0.01$, $\mathrm{p}<0.05$, as relative difference values $\triangle \mathrm{P} 2 \mathrm{P} 3$ compared with vector "zeros". 
indices, at least to distinguish states between rest and workload.

\subsection{Selection of ECG Indices to Distinguish States between Rest and Workload}

The percent of individual subject to all subjects whose indices had significant difference between rest and workload states is shown in Table $\mathbf{3}$, which is defined as the coincidence rate (CR). There were 4 indices whose coincidence rate were bigger than $85 \%$ at significant difference level ( $\mathrm{P}<0.05$ ), which contained indices of SdQR/SdRS, AgQ, AgS and AgS/AgQ in triangle QRS. Furthermore, the coincidence rate of AgS/AgQ was $100 \%$ at significant difference level $(\mathrm{P}<0.05)$.

\section{Discussion}

Angles of $\mathrm{Q}, \mathrm{R}$ and $\mathrm{S}$ are determined partially by the ratio between time scale and amplitude scale. So are angles of T wave triangle. Set $1 \mathrm{~mm}$ represents $0.1 \mathrm{mV}$ of $\mathrm{y}$ coordinates in ECG paper, and $0.04 \mathrm{~s} / \mathrm{mm}, 0.01 \mathrm{~s} / \mathrm{mm}$ and $0.005 \mathrm{~s} / \mathrm{mm}$ in $\mathrm{x}$ coordinates represents ECG paper speed of $25 \mathrm{~mm} / \mathrm{s}, 100 \mathrm{~mm} / \mathrm{s}$ and $200 \mathrm{~mm} / \mathrm{s}$, respectively. The morphology of triangle QRS and triangle $\mathrm{T}$ are shown in Figure 4. The effects of the proportion between time scale and amplitude scale are shown in Table 4 by the first heartbeat of Figure 4(a). The higher time resolution, the smaller AgQ but bigger AgR and AgS in triangle QRS, and the smaller AgTb and AgTb but bigger AgTp in triangle $\mathrm{T}$ of the first heartbeat in Figure 4. Particularly, both AgQ and AgTb transformed from obtuse angles to acute angles, but AgTp transformed form an acute angle to an obtuse angle in Table 4 . The time scale was chosen as $0.005 \mathrm{~s} / \mathrm{mm}$ to make the angle $\mathrm{R}$ in triangle QRS more clearly, i.e., $F_{T m}$ is 0.005 and $F_{A m}$ is 0.1 in Equation (4).

Table 3. Indices coincidence rates which had significant difference between rest and workload.

\begin{tabular}{ccccc}
\hline No & Index & $\begin{array}{c}\text { CR at } \mathrm{P}<0.05 \\
(\%)\end{array}$ & $\begin{array}{c}\mathrm{CR} \text { at } \mathrm{P}<0.01 \\
(\%)\end{array}$ & $\begin{array}{c}\mathrm{CR} \text { at } \mathrm{P}<0.001 \\
(\%)\end{array}$ \\
\hline 1 & TmRR & 100 & 100 & 96.67 \\
2 & SdQS & 70.00 & 66.67 & 63.33 \\
3 & SdQR/SdRS & 93.33 & 86.67 & 86.67 \\
4 & AgQ & 96.67 & 96.67 & 96.67 \\
5 & AgS & 86.67 & 86.67 & 80.00 \\
6 & AgS/AgQ & 100 & 96.67 & 96.67 \\
7 & LnQR & 66.67 & 60.00 & 43.33 \\
8 & SdTpTn & 83.33 & 73.33 & 63.33 \\
9 & PmTirTw & 70.00 & 50.00 & 46.67 \\
10 & ArTirTw & 66.67 & 46.67 & 43.33 \\
11 & LnTbTp & 76.67 & 70.00 & 63.33 \\
\hline
\end{tabular}
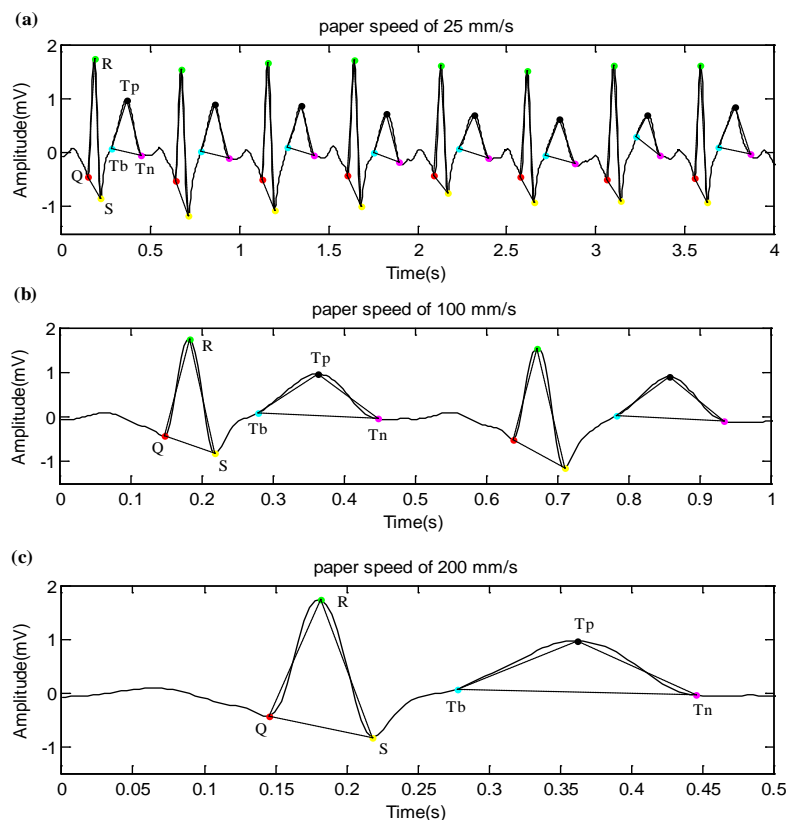

Figure 4. Effects of proportion between time scale and amplitude scale.

Table 4. Influence of the ratio between time scale and amplitude scale for the first heartbeat of Figure 4.

(a) angles in triangle QRS

\begin{tabular}{ccccc}
\hline time scale & AgQ & AgR & AgS & sum \\
\hline $0.04 \mathrm{~s} / \mathrm{mm}$ & 154.4930 & 4.1225 & 21.3841 & 179.9996 \\
$0.01 \mathrm{~s} / \mathrm{mm}$ & 111.6323 & 16.3844 & 51.9829 & 179.9996 \\
$0.005 \mathrm{~s} / \mathrm{mm}$ & 89.6826 & 32.1239 & 58.1931 & 179.9996 \\
\multicolumn{5}{c}{ (b) angles in triangle T } \\
time scale & AgTb & AgTp & AgTn & sum \\
$0.04 \mathrm{~s} / \mathrm{mm}$ & 93.7415 & 24.3581 & 61.8999 & 179.9996 \\
$0.01 \mathrm{~s} / \mathrm{mm}$ & 52.3109 & 81.5751 & 46.1135 & 179.9996 \\
$0.005 \mathrm{~s} / \mathrm{mm}$ & 31.2402 & 119.7902 & 28.9692 & 179.9996
\end{tabular}

Note: amplitude scale is $0.1 \mathrm{mV} / \mathrm{mm}$.

Compared with the predominance of parasympathetic tone during relaxation period, sympathetic tone predominates during exercise period and increases the cardiovascular workload and increases heart rate. He et al. [10] found that subjects with a higher HR response to exercise tend to have smaller abrupt changes in $\mathrm{R}$ wave amplitude (RWA) of lead CM at the interphases between rest and walking, as the gradual decrease in RWA during walking may be related to a gradual increase in HR and a gradual decrease in systemic peripheral resistance, and the gradual increase in RWA after walking may be related to a gradual decrease in HR and a gradual increase in systemic peripheral resistance. It is likely that the process of ventricular depolarization may not be fully accomplished because of the limited time of ventricular depolarization 
during exercise. The process of ventricular repolarization may not be fully accomplished too, because of the limited time of ventricular repolarization during exercise. So $\mathrm{T}$ wave may become smaller during exercise than during rest period. This hypothesis explained the reason why all side lengths, high lines, areas and perimeters in triangle $\mathrm{T}$ became smaller during workload than rest state. During recovery period after exercise, vagal reactivation resulted in increased parasympathetic tone and a declined in heart rate [1], which induced R-R interval increased and size of $\mathrm{T}$ wave increased when comparing with the exercise period in Table 2.

In this paper, the capability of the indices extracted from triangle QRS and triangle $\mathrm{T}$ was tested for distinguishing states between exercise and relaxation, and 4 characters from triangle QRS had significant differences (t test, $\mathrm{p}<0.05$ ) for distinguishing between exercise state and relaxation state for over $85 \%$ of subjects, which were: the ratio of $\mathrm{QR}$ side length to $\mathrm{RS}$ side length in triangle $\mathrm{QRS}$, the angle $\mathrm{S}$ and the angle $\mathrm{Q}$ as well as the ratio between them. Moreover, the ratio of angle $S$ to angle $\mathrm{Q}$ had significant differences ( $\mathrm{t}$ test, $\mathrm{p}<0.05$ ) for all subjects. ECG morphological indices performed very well at finding out the ECG changes that induced by exercise. Thus, they may aid in the noninvasive evaluation of physical stress levels. As a result, it is concluded that ECG waveform contains plenty of information in distinguishing states between exercise and relaxation.

\section{Acknowledgements}

This work was supported in part by Advanced Space Medico-Engineering Research Project of China (SJ201006, 2011SY5407019, 2012SY54B0601) and State Key Laboratory of Space Medicine Fundamentals and Application, China Astronaut Research and Training Center (SMFA12B09).

\section{REFERENCES}

[1] J. P. Higgins and J. A. Higgins, "Electrocardiographic Exercise Stress Testing: An Update beyond the ST Segment,” International Journal of Cardiology, Vol. 116, 2007, pp. 285-299.

http://dx.doi.org/10.1016/j.ijcard.2006.04.047
[2] Y. J. Li, H. Yan, W. Chen, L. Zhang and B. Zhang, "Different Characteristics of Heart Rate Variability in Mental and Physical Fatigue States," Space Medicine \& Medical Engineering, Vol. 23, No. 3, 2010, pp. 157-162.

[3] A. Svensbergh, M. Johansson, O. Pahlm and L. H. Brudin, "ST-Recovery Loop of Exercise-induced ST Deviation in the Identification of Coronary Artery Disease: Which Parameters Should We Measure?” Journal of Electrocardiology, Vol. 37, No. 4, 2004, pp. 275-283. http://dx.doi.org/10.1016/j.jelectrocard.2004.07.012

[4] Y. J. Li, H. Yan and Z. L.Wang, "Study of ElectrocardioWaveform Variability," Science in China, Series C: Life Sciences, Vol. 39, No. 12, 2009, pp. 1181-1187.

[5] H. Yan and Y. J. Li, "Electrocardiogram Analysis Based on the Karhunen-Loève Transform,” In: W. C. Yu, M. Zhang, L. P. Wang and Y. B. Song, Eds., 2010 3rd International Conference on BioMedical Engineering and Informatics (BMEI2010), Institute of Electrical and Electronics Engineers, Yantai, 2010, pp. 887-890.

[6] J. Z. Song, H. Yan, Y. J. Li and K. Y. Mu, "Research on Electrocardiogram Baseline Wandering Correction Based on Wavelet Transform, QRS Barycenter Fitting, and Regional Method," Australasian Physical \& Engineering Science in Medicine, Vol. 33, No. 3, 2010, pp. 279-283. http://dx.doi.org/10.1007/s13246-010-0033-5

[7] Y. J. Li, H. Yan, F. Hong and J. Z. Song, "A New Approach of QRS Complex Detection Based on Matched Filtering and Triangle Character Analysis,” Australasian Physical \& Engineering Science in Medicine, Vol. 35, No. 3, 2012, pp. 341-356. http://dx.doi.org/10.1007/s13246-012-0149-X

[8] Q. H. Zhang, A. I. Manriquez, C. Médigue, Y. Papelier and M. Sorine, "An Algorithm for Robust and Efficient Location of T-Wave Ends in Electrocardiograms," IEEE Transactions on Biomedical Engineering, Vol. 53, No. 12, 2006, pp. 2544-2552.

http://dx.doi.org/10.1109/TBME.2006.884644

[9] J. Mateo, P. Serrano and R. Bailon, "ECG-Based Clinical Indexes during Exercise Test Including Repolarization, Depolarization and HRV," Computers in Cardiology, Vol. 28, 2001, pp. 309-312.

[10] J. F. He, Y. Kinouchi, H. Yamaguchi and H. Miyamoto, "Exercise-Induced Changes in R Wave Amplitude and Heart Rate in Normal Subjects," Journal of Electrocardiology, Vol. 28, No. 2, 1995, pp. 99-106. http://dx.doi.org/10.1016/S0022-0736(05)80280-8 